Communications in Physics, Vol. 26, No. 3 (2016), pp. 287-295

DOI: $10.15625 / 0868-3166 / 26 / 3 / 8943$

\title{
SCREENING OF A SMALL SPHERICAL MACROION BY OPPOSITELY CHARGED FLEXIBLE POLYELECTROLYTE
}

\author{
VU VAN QUYEN ${ }^{1}$, VU THI HAI YEN ${ }^{2}$ AND NGUYEN THE TOAN ${ }^{1,3, \dagger}$ \\ ${ }^{1}$ VNU Key Laboratory Multiscale Simulation of Complex Systems, \\ VNU University of Science, 334 Nguyen Trai Street, Thanh Xuan, Hanoi, Vietnam. \\ ${ }^{2}$ Faculty of Physics, VNU University of Science, \\ 334 Nguyen Trai Street Thanh Xuan, Hanoi, Vietnam. \\ ${ }^{3}$ School of Physics, Georgia Institute of Technology, \\ 837 State Street, Atlanta, Georgia 30332-0430, USA \\ ${ }^{\dagger}$ E-mail: toannt@hus.edu.vn
}

Received 26 November 2016

Accepted for publication 31 December 2016

\begin{abstract}
Electrostatic interactions play important roles in the assembly, the structure and the functions of many biological as well as soft matter systems. In the presence of a macro-ion in aqueous solution, oppositely charged molecules mobilize around it to screen out its electrostatic potential. In this paper, we focus on screening of small macroion by a flexible polyelectrolyte using Monte-Carlo simulation. It is shown that the condensation of the polyelectrolyte around the macroion shows a first order phase transition from a dense to a dilute concentration of monomers. The width of the condensed region increases with stronger screening or with smaller macro-ion charges. For small macro-ion charge, or when the Coulomb interactions are strongly screened, no complexation between the polyelectrolyte and the macro-ion happens. Additionally, long polyelectrolyte protrudes both tails and loops from the macroion. This is quite different from the case of large macroions where only tails appear. Our results can be used to explain various experimental trends.
\end{abstract}

Keywords: polyelectrolyte, macroion complexation, flexible polymer, electrostatic screening.

Classification numbers: 05.10.-a, 87.15.-v. 


\section{INTRODUCTION}

Electrostatic complexation of an immobile macroion by highly charged polyelectrolyte (PE) is a common motif in many biological and soft matter systems. For example, in the chromatin, negative DNA winds around a positive histone octamer to form a complex known as the nucleosome [1,2]. This complexation is mostly due to electrostatics. It is found that the amount of DNA charges is about $15 \%$ higher than the amount of histone charges, a so-called charge inversion phenomenon which happens in strongly charged biological and soft matter systems [3]. The complexation between nucleocapsid proteins (NC) with viral RNA in the human immunodeficiency virus (HIV) capsid is another example of such complexation [4]. It is found that RNA condensation by NC macroions play a critical role in the reverse transcription from viral RNA to viral DNA. When the HIV capsid enters the cytoplasm after membrane fusion, it takes sometime before the capsid is disintegrated. During this time, the NC-condensed RNA molecules are partially reversed transcribed into DNA. However, if one ruptures the capsid, NC macroions diffuses away, RNA cannot be condensed and the reverse transcription is stopped. PE-micelle systems also an example of PE-macroion complexation with charge inversion [5,6]. These and other examples have created many theoretical and experimental works on electrostatic complexation of polyelectrolyte, DNA, RNA,... with macroions (see [3,7] for review).

In this paper, we visit problem of flexible polyelectrolyte condensation around an oppositely charged macroions. In a previous work [8], the complexation between polyelectrolyte with large macroion was studied. It is shown that the PE winds around the macroion forming a lattice, which cause the PE to overcharge the macroion. Additionally, long PE can protrude tails from the macroion in other one tail or two tail configurations $[9,10]$. Here, we focus on the situation where the macroion is small enough such that only a few monomers can condense on the macrion. The configurational space of the PE is much larger (almost the same as that of single free PE). As a result, the PE configurational entropy is able to compete strongly with the electrostatic interactions. Using Monte-Carlo simulation, we show that there can be a binding-unbinding transition where, for very weak screening, the PE can diffuse away from the macroion to gain configurational entropy. Additionally, in the binding state, the PE can protrude not only tails but also loops from the macroion.

This article is organized as follow. In Sec. II, we describe about the detail of simulation model and method. In Sec. III, the result of the Monte-Carlo simulation is presented and discussed. We conclude in Sec. IV.

\section{SIMULATION MODEL AND METHOD}

In this work, we use Monte Carlo computer simulation method [11] to study the complexation of $\mathrm{PE}$ and macroion. The polyelectrolyte is modeled as a chain of $N$ freely jointed hard spherical beads each with charge $e$ and radius $r_{b}=0.2 l_{B}$. The bond length of the PE is fixed and equal to $l_{B}$. Here $l_{B}=e^{2} / 4 \pi \varepsilon_{0} \varepsilon_{r} k_{B} T$ is the so-called Bjerrum length [12], where $e$ is the elementary charge, $k_{B}$ is the Boltzmann constant, $T$ is the absolute temperature in Kelvin, $\varepsilon_{r}$ is the relative dielectric constant of the medium and $\varepsilon_{0}$ is the vacuum permittivity. It is the length at which the Coulomb interaction between two elementary charges $e$ in solution is equal to the thermal energy $k_{B} T$. In a water solution of dielectric constant $\varepsilon=78$ at room temperature $(T \approx 298 K), l_{B}=7.12 \AA$. The parameters of the PE chosen so that its linear charge density is equal to at the critical Manning 
threshold [13]. This value is typical for highly charged polyelectrolytes such as RNA and DNA molecules. The macroion is modeled as a positively charged spherical macro-ion with charge $Z e$ with radius $l_{B}$ and is immobilized at the origin.

All aqueous solutions in biological systems always contain ions such as $\mathrm{Na}^{+}, \mathrm{Cl}^{-}, \mathrm{K}^{+}, \ldots$. Those ions can terminate electric field lines, and therefore can screen out Coulomb interactions among charges. Free ions do more than reduce the overall amplitude of Coulomb interactions they change the shape of the potential energy, making it go to zero exponentially (rapidly) beyond a characteristic distance called the Debye screening length. Especially, in water solution with monovalent mobile ions such as $\mathrm{Na}^{+}, \mathrm{Cl}^{-}$the electrostatic potential of a charge $q$ is:

$$
V(r)=\frac{q}{4 \pi \varepsilon_{r} \varepsilon_{0} r} \exp \left(-\frac{r}{r_{s}}\right)
$$

here: $r_{s}$ is the so-called Debye-Hückel screening length. In solution with a concentration $c_{s}$ of monovalent salt, $r_{s}$ is given by:

$$
r_{s}=\sqrt{1 / 8 \pi l_{B} c_{s}}
$$

As we can see $r_{s}$ is inversely proportional to square root of the concentration. Higher concentration of mobile ions leads to smaller $r_{s}$ or stronger screening. For typical $100 \mathrm{mM}$ of monovalent salt $(\mathrm{NaCl}), r_{s} \approx 10 \AA$. Eq. (1) shows that, at large distance $r>r_{s}$, the Coulomb interaction between particles are strongly screened (the potential decays exponentially) and at small distance $r<r_{s}$, $\exp \left(-r / r_{s}\right) \approx 1$, the standard Coulomb interaction is recovered. This is Debye-Hückel screening phenomenon.

To simulate the system of PE and macroion, the libSimEngine is used. This is a multi-core CPU+ GPGPU accelerated physics simulation engine developed by one of the authors (NTT). This engine uses OpenMP and OpenCL extensions to scale computational works among different CPU and GPGPU cores. Both molecular dynamics and Monte-Carlo simulations are supported by this engine. In this work, we use the Monte-Carlo module of the engine.

To relax the PE configuration globally, the pivot algorithm is used (see Fig. ??) (see Ref. [6] and references therein). In this algorithm, in an attempted move, a part of the chain from a randomly chosen monomer to one end of the chain is rotated by a random angle about a random axis. Because the pivot move is very radical, while the fraction of accepted moves decreases very slowly with increasing $N$, this algorithm is known to be very efficient in exploring the phase space of the PE. In addition to the pivot moves, to relax the PE configuration locally, the flip algorithm is used (see Fig. ??). In this algorithm, a randomly chosen monomer is rotated by a random angle about the axis connecting its two neighbors (if it is one of the end monomers, its new position is chosen randomly on the surface of a sphere with radius $l_{B}$ centered at its neighbor). In the simulation, the number of pivot moves is chosen to be about $30 \%$ of the total number of moves. $2 \times 10^{4}$ Monte Carlo steps are run for each set of parameters $\left(Z, r_{s}\right)$ (one Monte Carlo step is defined as the number of elementary moves such that, on average, every particle is attempted to move once). The usual Metropolis algorithm is used to accept or reject a trial move. Two different PEs, one with 256 beads and another with 512 beads are studied in this work (typical number of nucleic in viral RNA). The initial configuration of the PE is chosen to be either a straight rod or a Gaussian coil. Our final result does not depend on the choice of the initial configurations. This suggests that our system is properly equilibrated. 


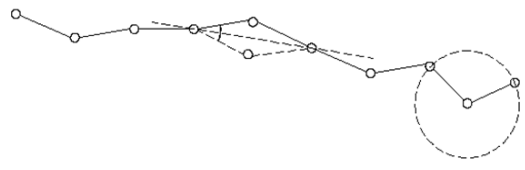

(a) Schematic flip MC move

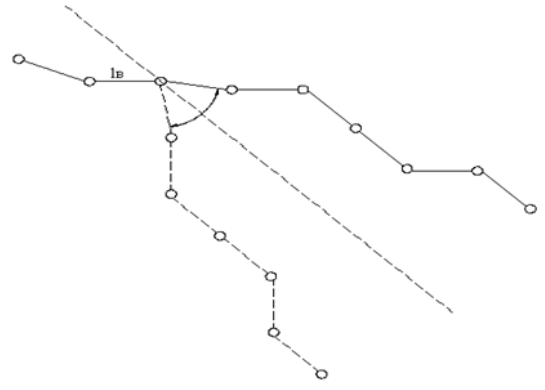

(b) Schematic pivot MC move

Fig. 1. Schematic figures showing two different Monte-Carlo moves that are used in the simulation. a) In a flip move to relax the polyelectrolyte configuration locally, a monomer is rotated by an random angle around the line connecting the neighboring monomers keeping all the bond lengths fixed. b) In a pivot move to relax the polyelectrolyte configuration globally, a part of the PE from a random monomer to one end is rotated by a random 3D angles centered around the chosen monomers.

\section{RESULTS AND DISCUSSIONS}

Our simulation result shows that depending on the set of parameter $\left(Z, r_{s}\right)$, there are 2 regimes of complexation. If the screening effect is strong (or at small $r_{s}$ ) or the macroion charge is small (small $Z$ ), no complexation between PE and macroion is observed. On the other hand, if the screening effect is weak (or at large $r_{s}$ ) or the charge of macroion is large (large $Z$ ), the Coulomb attraction to the macroion is very strong, so the PE remains within a smaller distance from the macroion.

To investigate whether the PE is forming a complexation with the macroion or not, the distance between the center of mass of the PE and the macroion at the origin is studied. The initial configuration of the PE is always around and centered at the macroion. The average distance of the breads from the immobile macroion located at the origin is defined as:

$$
D=\frac{\sum_{i}^{N} \sqrt{x_{i}^{2}+y_{i}^{2}+z_{i}^{2}}}{N}
$$

Here we just describe the detail when $N=256$. The bind-unbinding transition as function of the electrostatic coupling is similar for the case of $N=512$.

Examples of the behavior are shown in Fig. 2, for $Z=100$, where the distance in Eq. (3) is plotted as a function of MC steps. Panel (a) and (b) show the transition from no complexation to complexation. In Panel (a), with $r_{s}=0.8$, it is evident that the PE cannot be attracted to the macroion. In this no-complexation phase, the PE simply diffuses from the macroion at the origin and the average distance of the beads from the macroion go to $\infty$ as with increasing simulation time. In Panel (b), (c) where the distance is within tens of $l_{B}$ thus the PE form a complex with the macroion. With $r_{s}=0.95$, we recognize this is the phase transition value beyond which the PE is attracted to the macroion, at this value we see that $\mathrm{D}$ varies in interval $(10: 40) l_{B}$. For larger $r_{s}$ 
$\left(r_{s}=20\right.$, really large) the PE is more strongly be attracted, so the PE center vary in smaller interval $(15: 19) l_{B}$. This is because more PE monomers are captured with screening distance.

(a)

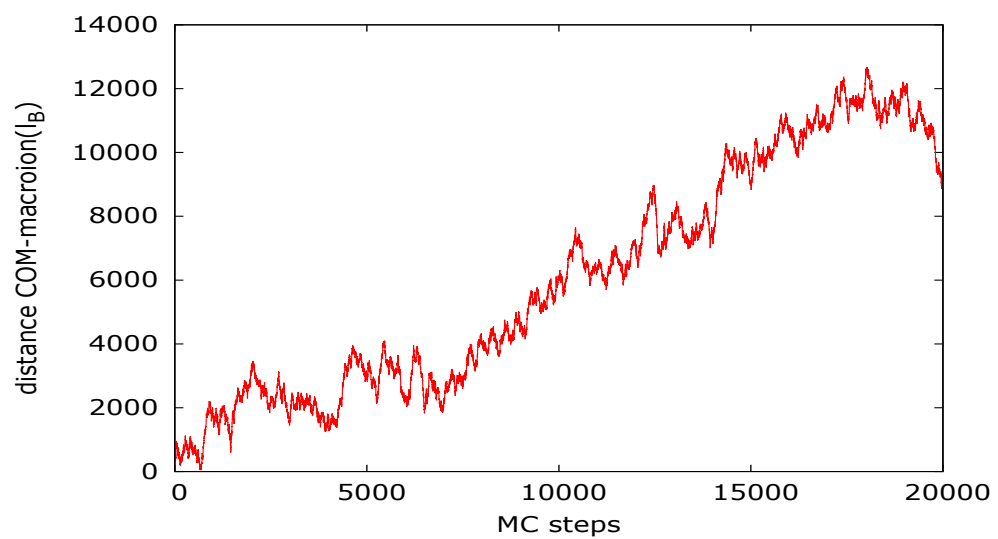

(b)
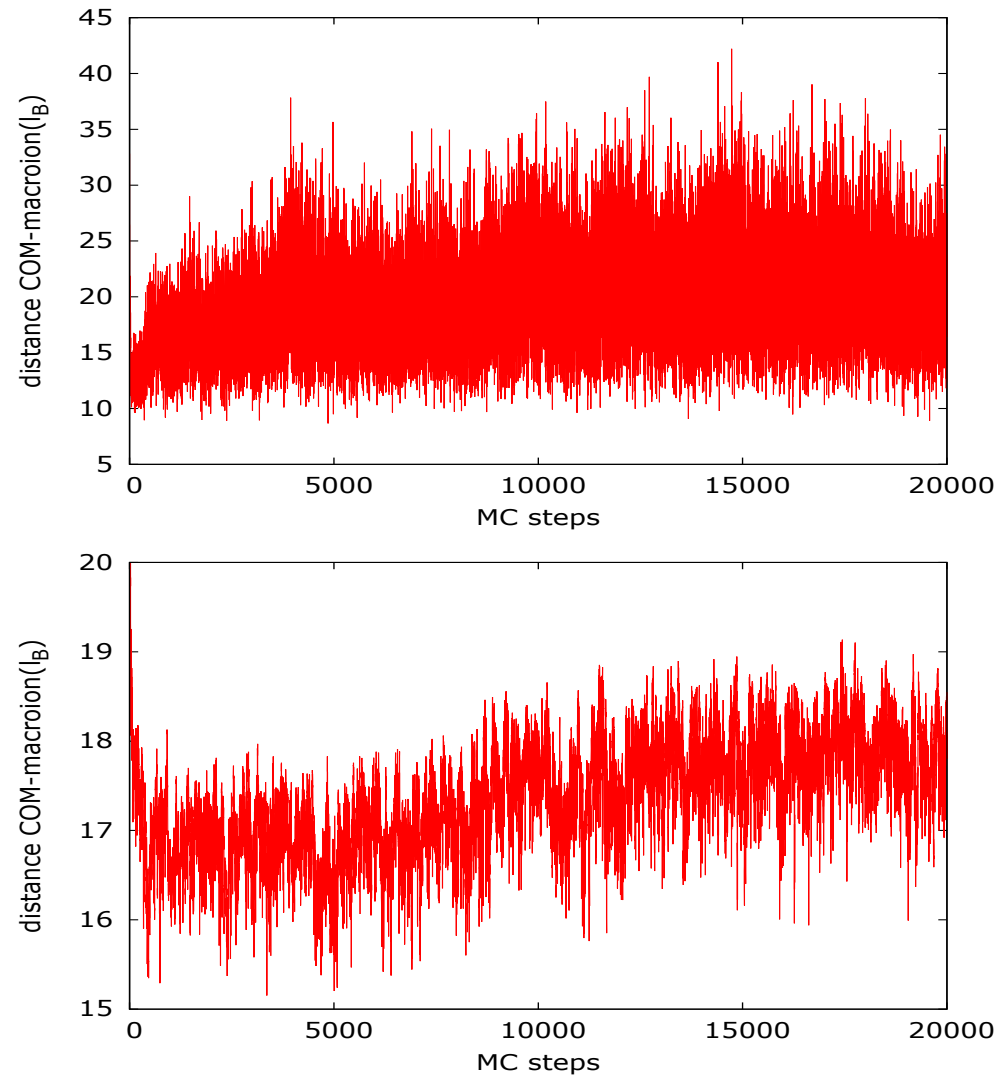

Fig. 2. The average distance of beads from the macroion as a function of MC steps is plotted for (a) $r_{s}=0.8$; (b) $r_{s}=0.95$ and (c) $r_{s}=20$ (for the same charge of protein $Z=100)$. Panel (a): The PE diffuses away. Panel (b),(c): The PE is remain within tens of $l_{B}$ from the macroion. 
Our main results are shown in Fig. 3, where we construct the phase diagram of complexation in the plane of the set of parameter $\left(Z, r_{s}\right)$. The upper-right region is complexation phase and the lower-left region is no-complexation phase. The complexation between the PE and the macroion is due to the competition between the configurational entropy of the PE and its electrostatic attraction to the macroion. Configurational entropy of the PE with 512 beads is larger than that of 256 beads, so it is harder to form complex with the macroion. As the result we can see that the phase boundary of the 512-beads PE is at higher values of $r_{s}$ than that of the 256-beads PE (notice the logarithmic scale the vertical axis in Fig. 3).

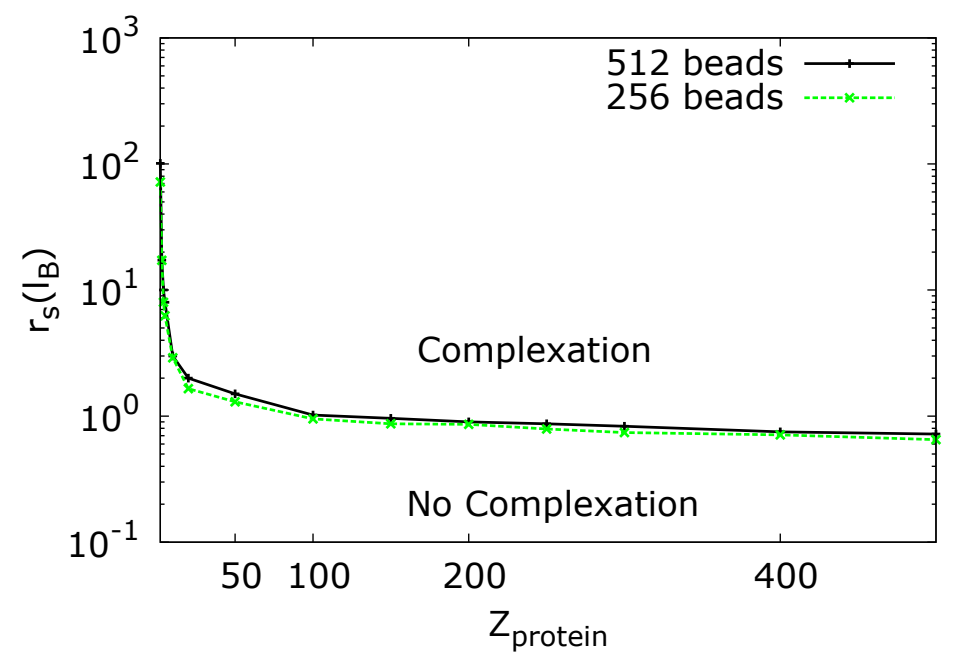

Fig. 3. Phase diagram (linear log scale)

From $Z=2$ to $Z=100$, the transition value $r_{s}$ rapidly decreases when $Z$ increases but from $Z>100$, this transition value $r_{s}$ almost does not decrease too much when $Z$ increases. For the case of $Z=1$, we find no value of $r_{s}$ that can complex the PE. This suggests that when $Z=1$, the PE cannot be attracted. The standard Coulomb potential has also been simulated with the corresponding to $r_{s}=\infty$ and strong electrostatic attraction. Even in this case, no complexation is observed. It is due to the interaction energy between macroion $(Z=1)$ and the PE is of comparable magnitude with $k_{B} T$, much smaller than the macroions entropy. It is also clear that the flexible polymer has larger linear charge density than the stiffer polymer, so if we have a macroion with $\mathrm{Z}$ $=1$ interacts with stiffer polymer, we also cannot observe the complexation.

In Fig. 4 we plot the concentration profile of monomer as a function of distance from the macroion for $Z=100$ with different values of $r_{s}$. In an other study, we study the same problem using mean-field theory for flexible polyelectrolyte. When $r$ is small, the concentration profile of monomer is very similar to the results of self-consistent mean-field theory, the concentration decays as exponentially with $r$. However, at larger $r$, the concentration profile deviates from mean-field theory. As $\rho(r)$ decrease when $r \rightarrow \infty$ and the PE is captured at a finite distance from the macroion. Mean-field theory also fails to predict the unbinding transition of PE-macroion complex. This is because mean-field theory treats the electrostatic attraction to the macroion as 


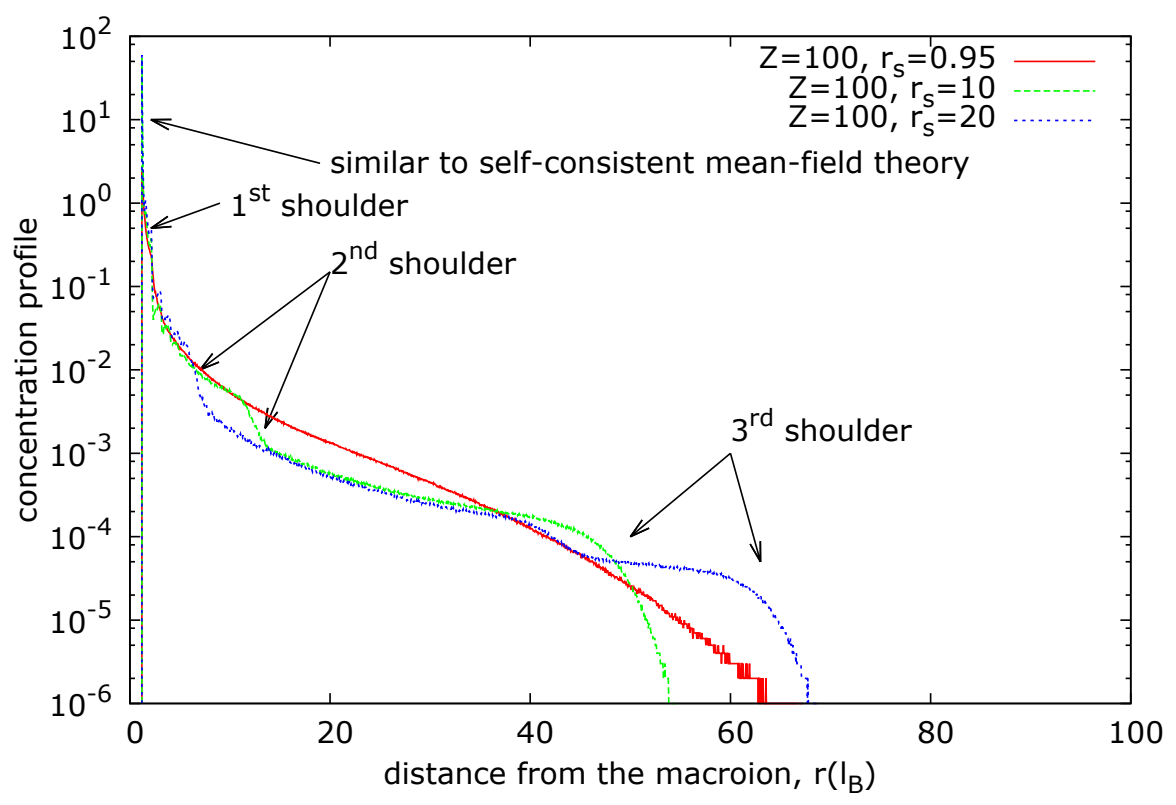

Fig. 4. Monomer density profile in the complexation phase for $Z_{\text {macroion }}=100$ with different screening length value.

the dominant term and the fluctuation of the monomer density is small. Clearly, this cannot capture the unbind limit.

Additionally, there are two or more shoulders observed where the monomer density, $\rho(r)$, experience rapid decreases with $r$. This can be easily understood. The complexation - no complexation is a first order phase transition, so the first shoulder correspond to first order transition from the dense (liquid) near the macroion surface to the dilute (gas) phase of monomers. However, for our small macroion, loops can also appear to maximize the PE configurational entropy. With larger $r_{s}$, the PE is attracted stronger so near the macroion the concentration of monomer is very large and far from macroion the concentration is small because the repulsive interaction of monomer-monomer is strong so we can see that phase transition with larger $r_{s}$ is more pronounced than the case with smaller $r_{s}$.

The physics behind second and next shoulder is more complicated. It relates to the fact that loops are protrude from the macroions surface. For large macroion, only tails of the PE are protrude from the surface [8-10]. To clearly demonstrate the appearance of loops in the PE configuration, in Fig. 5, we show a snapshot of our system. The left figure is for $Z=100$ and $r_{s}=$ 0.95 (this is the phase transition value corresponding to $Z=100$ ). The right figure is with $r_{s}=20$. Compared to the case of large macroion, it is evident that small macroion allow the PE to have more configurational entropy and induce loops into the system. Large macroion mostly allows for only one or two tails of the PE to protrude from the macroions. These can have implication in the condensation of PE by several small macroions. 

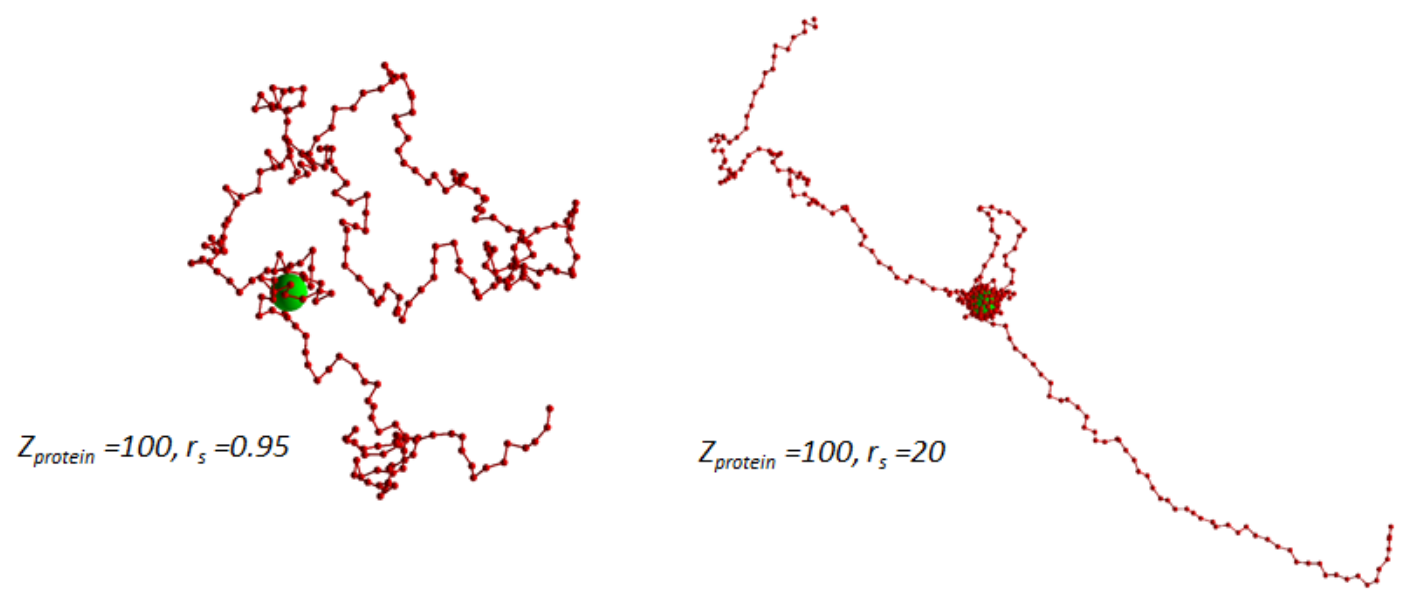

Fig. 5. Visualization of system when $r_{s}=0.95$ and $r_{s}=20$ for the same charge of macroion $(\mathrm{Z}=100)$. The phase transition when $r_{s}=20$ is more clear.

Our result gives a qualitative explanation for the experimental result of HIV NC-RNA complexation. The concentration of NC macroion inside the HIV virus capsid is important to condense RNA. If HIV capsid is ruptured before the reverse transcription stage is completed, $N C$ macroions diffuse away, $Z$ becomes very small as the result, the reverse transcription is stopped, thus no viral DNA can be formed.

\section{CONCLUSIONS}

In this work, the complexation between flexible polyelectrolyte with a small oppositely charged macroion is studied.

It is found that, depending on the charge of the macroion and depending on the strength of the electrostatic screening of the solution, the PE can or cannot form a complex with macroion. This phase transition is of the first order type. We obtain the phase transition diagram of the polyelectrolyte-macroion complexation as the function of the set of parameters $\left(Z, r_{s}\right)$.

In complexation phase, near the macroion, the PE behaves as if the excluded volume is not important. This is in agreement with mean-field theory. However, far away the self-consistent mean-field theory fails to predict the no-complexation phase. Therefore we do not observe the shoulder in field profile in mean-field theory. This is a known limitation of many mean-field theories since these theories ignore the correlation among the monomers. When the attraction to the macroion is strong, such correlations can be neglected. However, when the attraction to the macroion is weak, the correlations among monomers can be bigger than their attraction to the macroion. In our specific case, the mean-field theory fails to capture the behaviors of the PE in the strong screen case.

\section{ACKNOWLEDGMENT}

This work is supported financially by the Vietnam National Foundation for Science and Technology (NAFOSTED) under grant number 103.02-2012.75. 


\section{REFERENCES}

[1] K. Luger, A. W. Mäder, R. K. Richmond, D. F. Sargent and T. J. Richmond, Nature 389 (1997) 251-260.

[2] A. Johnson, B. Alberts, D. Bray, J. Lewis, K. Roberts and M. Raff, Molecular biology of the cell, 5th ed. ed., Garland Science, 2007.

[3] A. Y. Grosberg, T. T. Nguyen and B. Shklovskii, Rev. Mod. Phys. 74 (2002) 329.

[4] M. Götte, X. Li and M. A. Wainberg, Archives of Biochemistry and Biophysics 365 (1999) 199-210.

[5] Y. Wang, K. Kimura, Q. Huang, P. L. Dubin and W. Jaeger, Macromolecules 32 (1999) 7128-7134.

[6] T. Wallin and P. Linse, Langmuir 12 (1996) 305-314.

[7] H. Boroudjerdi, Y.-W. Kim, A. Naji, R. R. Netz, X. Schlagberger and A. Serr, Physics reports 416 (2005) $129-199$.

[8] T. T. Nguyen and B. I. Shklovskii, Physica A 293 (2001) 324.

[9] Park, S. Y., Bruinsma, R. F. and Gelbart, W. M., Europhys. Lett. 46 (1999) 454-460.

[10] Mateescu, E. M., Jeppesen, C. and Pincus, P., Europhys. Lett. 46 (1999) 493-498.

[11] M. P. Allen and D. J. Tildesley, Computer simulation of liquids, Clarendon Press, Oxford, 1987.

[12] N. J. Bjerrum, Z. Phys. Chem. (Munich) 119 (2000) 145.

[13] G. S. Manning, J. Chem. Phys. 51 (1969) 924. 